\title{
Secondary flows in slow granular flows
}

\author{
Peter Varun Dsouza ${ }^{1, \star}$, Krishnaraj K.P. ${ }^{1, \star \star}$, and Prabhu R Nott ${ }^{1, \star \star \star}$ \\ ${ }^{1}$ Indian Institute of Science, India
}

\begin{abstract}
Recent findings by Krishnaraj and Nott [1] show that a granular material sheared in a cylindrical Couette cell at low shear rates forms a single secondary vortex. The vortex spans the entire width of the Couette cell and has a sense opposite to the centrifugally driven Taylor-Couette vortex in a Newtonian fluid - it is in fact shown to be driven by shear-induced dilation. Krishnaraj and Nott [1] show that the vortex also explains a rheological anomaly observed earlier [2], wherein all components of the stress on the outer cylinder increase nearly exponentially with depth from the free surface. In this study, we test the robustness of this vortex by varying the parameters of the grain contact model. We show that the presence of a free surface is not essential for the formation of the secondary vortex. The vortex forms even when a rigid plate of finite weight confines the granular column at the top. We find that as the shear rate is increased, an additional centrifugally-driven vortex appears. This new vortex keeps growing until, at Savage number close to one, the dilation-driven vortex disappears. We also present the variation of the wall stresses at the inner cylinder with depth. Finally, we argue that the secondary flow can also help to understand the rheological behaviour observed in geometries such as the split-bottom Couette device [3]
\end{abstract}

\section{Introduction}

The cylindrical Couette device (Figure 1) is a standard setup used for measurement of the rheological behaviour of fluids. However, adapting the Couette apparatus to study particle flows is not straightforward. Gutam et. al. [2] have shown that on shearing the material, all components of the stress along the outer wall increase exponentially from the free surface. They also show that the component of the shear stress in the vertical direction of gravity changes sign upon commencement of shear. Krishnaraj and Nott [1] showed the presence of a system-spanning secondary vortex in this geometry and showed that it explains the stress anomaly (Figure 2). Understanding such a flow is crucial in developing continuum models for granular flows. In this paper we test for the robustness of this secondary flow. We do this by varying the simulation parameters. We also show how the flow behaves under load or high shear rates.

\section{Methods}

Granular flows can be classified into slow, intermediate and rapid flows. This is given by the Savage number $S a$, the ratio of inertial stress to the system stress scale. The Savage number is related to the Inertial Number, $I$, which are given by

$$
S a=I^{2}=\frac{\rho g W}{N}
$$

\footnotetext{
${ }^{\star}$ e-mail: varun@chemeng.iisc.ernet.in

$\star \star \mathrm{e}$-mail: krishnaraj@chemeng..iisc.ernet.in

$\star \star \star$ e-mail: prnott@chemeng.iisc.ernet.in
}

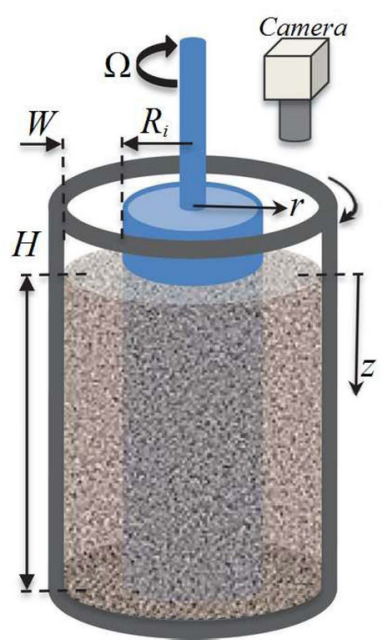

Figure 1: A schematic of the cylindrical Couette.

where $\rho$ is the particle density, $W$ is the system length scale and $N$ is the stress scale. Rapid flows are given by $S a \sim 1$. In most of our studies, the inner cylinder is rotated with a shear rate $\dot{\gamma}$, which corresponds to a Savage number of order $10^{-6}$. For higher shear rates, refer to Section 4 .

The Couette device used in the experiments is a custom built setup. It is filled with particles to a height, $H$, of $27 \mathrm{~cm}$. The annular gap, $W$, used in this paper is $4 \mathrm{~cm}$. We measured the stress along the outer wall for glass beads (bulk density, $\rho=1600 \mathrm{~kg} / \mathrm{m}^{3}$, average diameter $d_{p}=$ $0.833 \mathrm{~mm})$ and for mustard seeds $\left(\rho=720 \mathrm{~kg} / \mathrm{m}^{3}, d_{p}=1.2\right.$ $\mathrm{mm})$. We measured the stress profile along the outer wall 


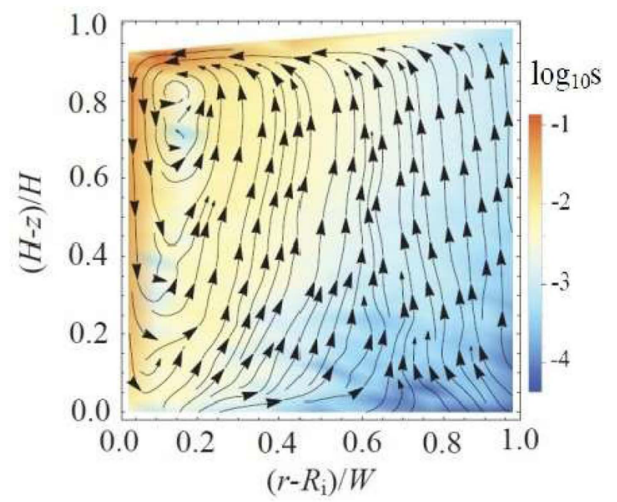

Figure 2: Streamlines of the secondary flow at steady state for a fill height $30 d_{p}$. The background colour is $\log _{10} s$ where $s \equiv\left(v_{r}^{2}+v_{z}^{2}\right) / V_{W}$, is the magnitude of the secondary flow. $V_{W}$ is the velocity of the inner cylinder surface. [1]

and the velocity profile of the particles at the top surface. The velocity profile was obtained by video imaging the top surface and carrying out Particle Image Velocimetry (PIV) analysis using the PIVlab MATLAB toolbox [4]. The ring used to confine the top surface had a window made of optical glass for imaging. The simulations method was the same as in [1].

\section{Effect of confinement with a load}

\subsection{Stress profile}

We tested to see if the presence of a free surface is important for the formation of the secondary flows. To test this, we placed a freely floating ring in the annular gap of the Couette. This ring is allowed to move freely along the $\mathrm{z}$ direction but restricted along $r$ and $\theta$. This is done such that the weight of the ring falls only on the granular material. This also allows the material to dilate freely. Figure 3 shows the normal stress, $\sigma_{r r}$, and shear stress, $\sigma_{r z}$. We still see the exponential rise of $\sigma_{r r}$, and that $\sigma_{r z}$ has a sign opposite to a static column.

\subsection{Kinematics}

The exponential decay of the tangential velocity, $V_{\theta}$, is a characteristic of slow particle flows in a Couette. The inward movement of particles with velocity, $V_{r}$, along the top surface is necessary to close the vortex. With a confinement, the top surface is no longer traction free making it more difficult to close the vortex. Figures 4(a) and 4(b) shows how the velocity profile changes under confinement. The material forms a slope on the surface to some distance from the inner cylinder up on reaching steady state. Without confinement, the material slopes all the way till the outer wall. The particles close to the outer wall no longer move inward because of the applied traction. How far the material slopes is dependent on the confining weight. The particles that lie on this slope are not restricted by the confinement's traction and can flow inward. (a)

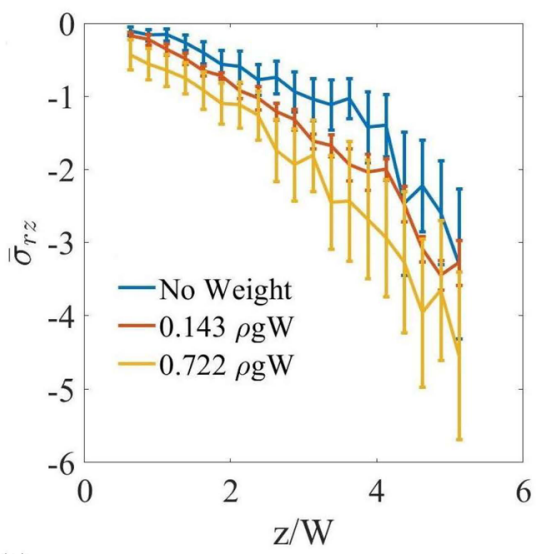

(b)

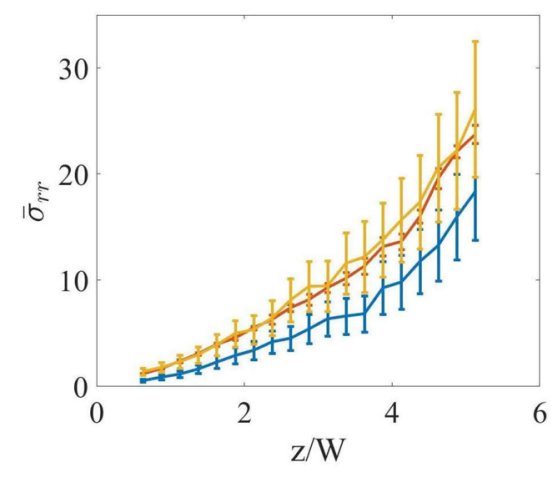

Figure 3: (a) Shear and (b) normal stress on the outer wall of a sheared, cylindrical Couette. The stresses, $\bar{\sigma}$, are scaled by $\rho g W$ and the distances are scaled by the Couette gap, $W$. Each data point is represented as mean \pm standard deviation.

\section{Varying the simulation model parameters}

The model used for the DEM simulations was proposed by Silbert et. al. [5]. This model assumes that the normal contact between particles can be represented by a linear spring with elastic constant $k$ and a dash-pot with a damping constant $\gamma$ in parallel. The tangential contact is similar but has a Columbic slider with coefficient $\mu$ to represent friction in series with the parallel, spring and dash-pot system. Consider two particles $i$ and $j$. The normal force, $F_{n}$, and tangential force, $F_{t}$, between them are

$$
\begin{gathered}
\mathbf{F}_{n}^{(i)}=k_{n} \delta \mathbf{n}-m_{e f f} \gamma_{n} \mathbf{v}_{n} \\
\mathbf{F}_{t}^{(i)}= \begin{cases}-k_{t} \Delta \mathbf{s}-m_{e f f} \gamma_{t} \mathbf{v}_{t} & \text { if }\left|F_{n}^{(i)} / F_{t}^{(i)}\right|<\mu \\
-\mu\left|F_{n}^{(i)}\right| \mathbf{v}_{\mathbf{t}} & \text { otherwise }\end{cases}
\end{gathered}
$$

Here, $\delta$ is the overlap between the particles, $\mathbf{n}$ is the unit normal joining the two centers in the direction of $i, m_{e f f} \equiv$ $\left(1 / m_{i}+1 / m_{j}\right)^{-1}$ is the effective mass, $v_{\mathbf{n}}$ and $\mathbf{v}_{\mathbf{t}}$ are the normal and tangential relative velocities at contact and $\Delta \mathbf{s}$ is the tangential displacement.

In the following subsections we show that the secondary flow is not dependent on the model parameters 
(a)

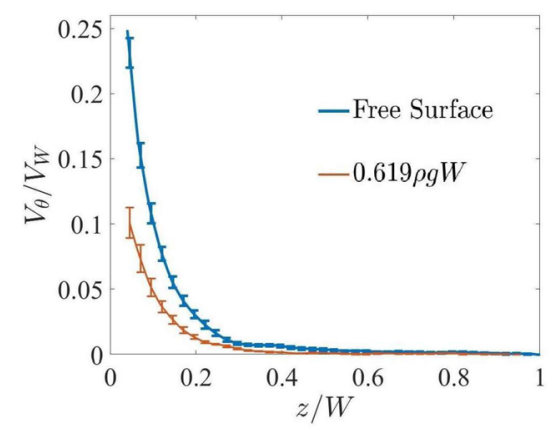

(b)

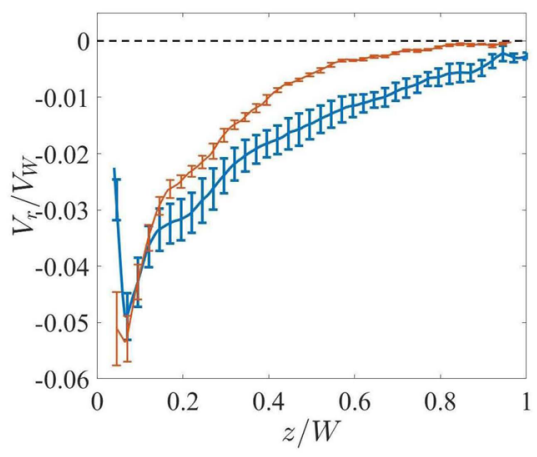

Figure 4: (a) Tangential and (b) radial velocity profiles along the top surface with and without confinement. The velocities are scaled by the inner cylinder velocity, $V_{W}$, and the distances are scaled by the Couette gap, $W$. The error bars are one standard deviation in length.

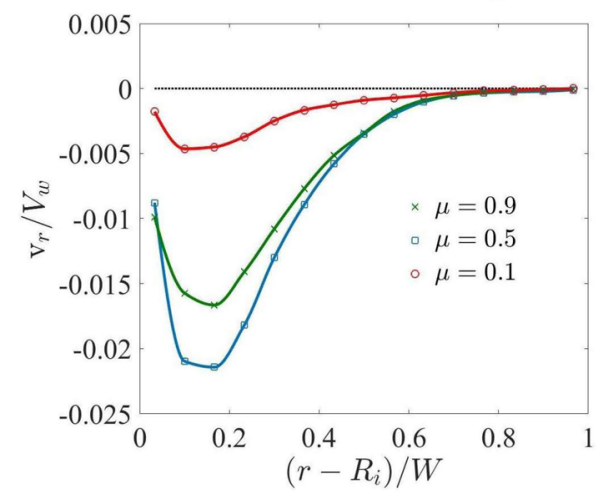

Figure 5: Radial velocity along the free surface for different values of $\mu$

used. We vary $\mu$ and $\dot{\gamma}$ in this paper. The damping coefficient $\epsilon$ is related to the restitution coefficient by Equation 3. We found varying $\epsilon$ from 0.4 to 0.9 in steps of 0.1 had no change in our results. Krishnaraj and Nott have shown how $k$ affects the system in [1].

$$
\epsilon_{n, t}=\exp \left(-\gamma_{n, t} t_{c o l} / 2\right)
$$

where $t_{c o l}$ is the collision time given by

$$
t_{c o l}=\pi\left(2 k_{n} / m-\gamma_{n}^{2} / 4\right)^{-1 / 2}
$$
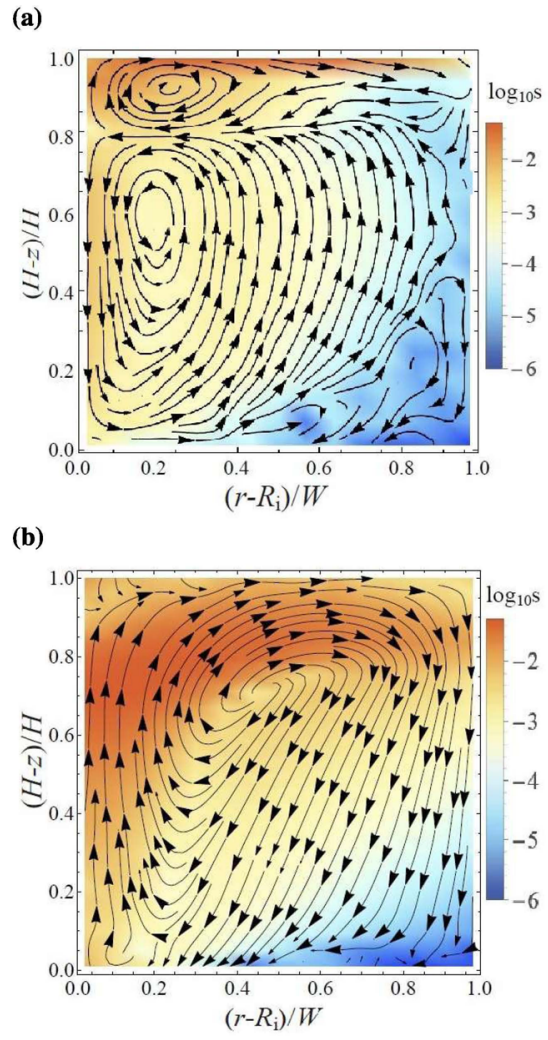

Figure 6: Streamlines for (a) $S a=2 \times 10^{-2}$, (b) $S a=1$.

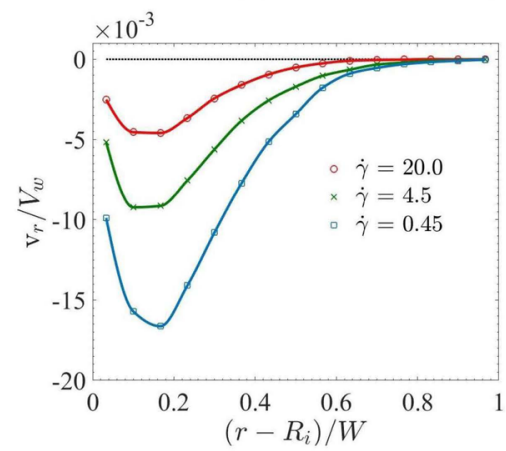

Figure 7: Radial Velocity at the top surface for different shear rates.

Figure 5 shows how the radial velocity profile changes along the top surface of the material. We can see how changing the value of $\mu$ does not change the direction of $v_{r}$, the radial velocity. The secondary flow is a characteristic of slow and intermediate flows. Increasing the shear rate, $\dot{\gamma}$ from $0.45 s^{-1}$ in the slow flow regime $\left(S a=2 * 10^{-6}\right.$ ) (Figure 2$)$ to the inertial regime ( $S a \equiv 1)$ replaces the dilationdriven vortex with a centrifugal vortex (Figure 6).

\section{Stress on the inner cylinder}

Gutam et. al. [2] have reported the stress exerted on the outer wall of the Couette. Figure 8 shows the normal stress on the inner cylinder obtained from the DEM simulations. We can see that even here, there is an exponential increase in the normal stress with respect to depth from the free 


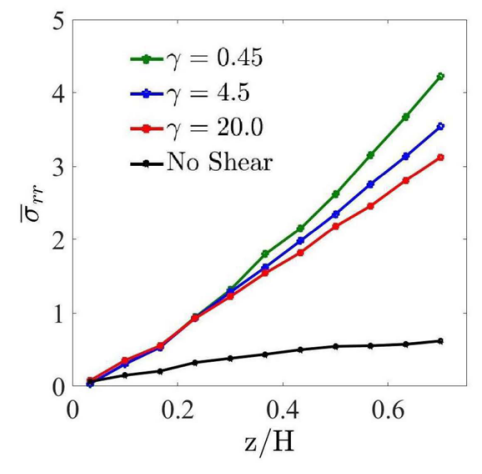

Figure 8: Normal Stress along the inner cylinder for different shear rate and for a static column.

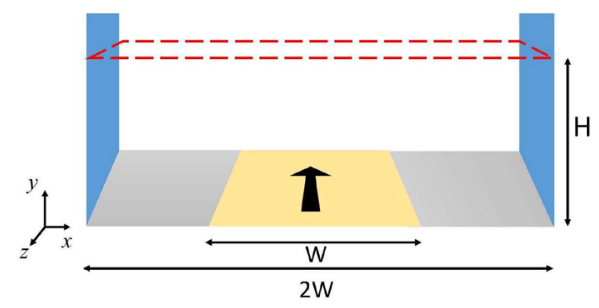

Figure 9: Schematic of a Cartesian equivalent of the splitbottom Couette.

surface. The stress along the vertical axis of inner cylinder was computed using DEM simulations. The radial wall stress profiles show similar exponential rise like the outer wall radial stress. Interestingly the sign of inner wall axial stress also changes after initiation of shearing the material. The direction of the radial vector pointing from the material to wall is opposite to the case of the outer wall. Hence the traction imposed by the material on the wall in both cases push the walls upwards against the direction of the gravity vector. Our results here further extend the that the anomalous wall stress profiles on the outer wall reported in previous study is true for the inner wall of the Couette as well.

\section{Other systems with secondary flows}

Secondary vortex formation has been seen in other studies $[6,7]$ though none of them could discern their cause. Further work could to characterize the vortex build-up using techniques similar to those suggested by [8]

The secondary vortex is dilation-driven, so we expect to see similar flows in other geometries. To test this, we carried out DEM simulations of the geometry shown in Figure 9. This is a box with periodic walls in the $\mathrm{x}$ and $\mathrm{z}$ directions. The bottom wall consists of two parts. The yellow section moves with a velocity $10 \sqrt{g d_{p}}$ in the $-z$ direction. The grey sections are stationary. Gravity acts along $-y$. This is a Cartesian equivalent of the split-bottom Couette used in [3].

We pour particles up to a height of $H$. At steady state, we get the velocity profile shown in Figure 10. These are the streamlines seen in the $x-y$ plane, averaged over the depth of the system. Here as well, we can see flows similar to those seen in the Couette. This shows that the secondary flow we obtain is not an artefact of the Cylindrical Couette. Further work is needed to understand these results.

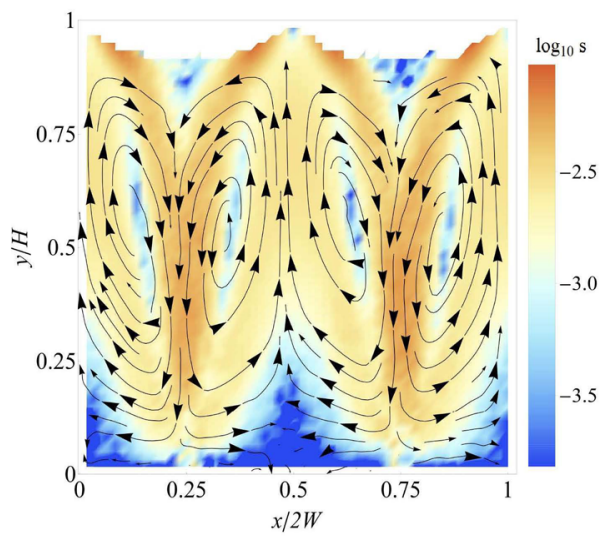

Figure 10: Streamlines obtained in the split-bottom Couette

\section{Conclusion}

We have tested and shown that the secondary vortex in a Couette still persists despite changes in the model parameters. We also tested the robustness of the flow under a confining weight and in the absence of a traction free surface. Finally, we also showed that such flows persist in other geometries too.

\section{References}

[1] Krishnaraj K.P., P.R. Nott, Nat. Commun. 7, 10630 (2016)

[2] K.J. Gutam, V. Mehandia, P.R. Nott, Phys. Fluids 25.7, 070602 (2013)

[3] K. Nichol, M. van Hecke, Phys. Rev. E 85.6, 061309 (2012)

[4] W. Thielicke, E.J. Stamhuis, J. Open Res. Software, 2.1, (2014)

[5] L.E. Silbert, et al., Phys. Rev. E, 64, 051302 (2001)

[6] R. Khosropour, J. Zirinsky, H.K. Pak, R.P. Behringer. Phys. Rev. E, 56.4 4467 (1997)

[7] N. Murdoch, et al. Phys. Rev. Lett. 110.1, 018307 (2013)

[8] P.G. Rognon, T. Miller, B. Metzger, I. Einav, Granular Matter 17.2 177-188 (2015) 